\title{
2050: The Year of Our Carbon-Neutral Food System
}

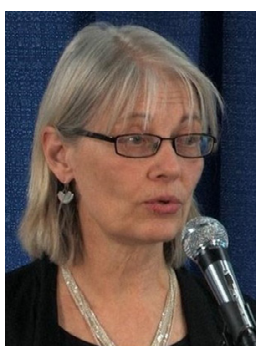

It is unequivocal that human influence has warmed the atmosphere, ocean and land. Widespread and rapid changes in the atmosphere, ocean, cryosphere and biosphere have occurred. ${ }^{1}$

This is the leading conclusion in the first United Nations Intergovernmental Panel on Climate Change (IPCC) working group report as part of the Sixth Assessment released on August 9. As science professionals, we understand the level of evidence necessary for the use of "unequivocal," and this assessment presents the latest advances in climate change science with a deeper understanding of the relationship of specific levels of carbon dioxide, methane, and other greenhouse gases with physical changes on our planet, including extreme climate events-heat waves, heavy rainfall, and droughts - that are more frequent and severe. ${ }^{1}$ The disruption affects not only humans but all other life on the planet, including plants and animals.

The necessary action was emphatically repeated throughout the IPCC press conference; there must be "rapid, strong and sustained reductions in emissions." ${ }^{2}$ Fossil fuel burning, the main source of our rising carbon dioxide levels must rapidly decline in the next two decades. Reductions of all greenhouse gases including carbon dioxide, methane and nitrous oxide must reach net zero emissions by 2050, if we are to limit global warming to $1.5^{\circ} \mathrm{C} .^{1}$ For the first time, the IPCC report details regional changes under different levels of GHG changes and the subsequent rise in temperature to provide the basis for immediate, appropriate actions necessary for resilience and mitigation.
To stop fossil fuel burning and reach net-zero GHG emissions by 2050 requires a methodical approach that involves all people across the planet. However, immediate, consistent individual change is necessary because every fraction of greenhouse gas (GHG) emission released each day will compromise our future. Meerow and Woodruff ${ }^{3}$ suggest 7 principles for strong climate change planning, which we can consider as we plan: set ambitious, yet attainable goals; provide a strong fact base with the best available data; outline diverse strategies to achieve goals; engage the public and foster justice in all planning processes; coordinate efforts to address climate change across actors, sectors, and plans; include a clear process for implementation and monitoring; and address climate change uncertainty.

The global food system, including food loss and waste emits $21 \%$ to $37 \%$ of the total global GHG emissions. ${ }^{4}$ High-input, resource-intensive farming systems have high levels of GHG emissions and cannot deliver sustainable food and agricultural production. While GHG emissions are produced at every step of the food system from farm to table, dominant food systems differ by nation and region resulting in different contributions to the climate change crisis. A lifecycle assessment of GHG emissions from U.S. household food choices found that 899 billion kilograms of $\mathrm{CO}_{2}$ emissions were generated from food spending in 2013. ${ }^{6}$ Of this, $67.9 \%$ was from production and manufacturing, 25.4\% from restaurant and retail sectors, and $4.7 \%$ from truck transportation. Weekly $\mathrm{CO}_{2}$ emissions per household were highest for red meat, fresh vegetables and melons, cheese, other milk products, and poultry. Identifying areas of greatest $\mathrm{GHG}$ emissions for different food systems will be necessary to develop the diverse strategies needed to reach our goal.

Increasingly, household food systems are dependent on national and global food systems, as we experienced with the COVID-19 pandemicrelated food system disruptions; therefore, a global food system approach will avoid climate mitigation in one area of the food system that would be offset by increased emissions or loss of carbon sinks in another area, while supporting our biodiversity and natural resource base. Rosenzweig and colleagues ${ }^{4}$ advocate for a global food system approach and list responses in different stages of the food system that synergistically support mitigation and adaptation while providing benefits to livelihoods, health, water, biodiversity, and reduced pollution. These responses include changes both in the supply side of improved plant and animal production and management, as well as demand-side responses such as dietary change, reduction of food loss, and reduction of food waste. ${ }^{4}$

These changes align with a recent analysis by Clark and colleagues $^{7}$ that modeled 5 strategies to reduce global food system emissions to achieve the Paris Agreement climate change targets of limiting $1.5^{\circ} \mathrm{C}$ and $2^{\circ} \mathrm{C}$ above preindustrial levels. The five strategies include the following: globally adopt a plant-rich diet; adjust global per capita caloric consumption to healthy levels; achieve high yields by closing yield gaps and improve crop genetics and agronomic practices; reduce food loss and waste by $50 \%$; and reduce the GHG intensity of foods by increasing efficiency of production. All 5 strategies would need to be implemented $100 \%$ to achieve the 2050 target; no single strategy would be successful.

This climate crisis demands immediate attention and significant sustained action. Our participation in developing a long-term strategy for food system change is necessary, and action can begin with knowing our regional food system. Which regional 
farmers incorporate or are transitioning to more sustainable production models, such as agroecology, silvapasture, and agroforestry? Does your region have policies to maintain access to affordable land, especially for small- and medium-holder farms? Can supply chains to retail, restaurants, and institutions be reduced? Do local laws support urban green spaces for gardens and foraging? Are there incubator spaces with certified kitchens available for entrepreneurs to develop added-value food products that help reduce food loss and waste? Do restaurants, schools, and community events celebrate with plant-based menus utilizing regionally sourced foods? Is food waste monitored in your community? We need daily effort to reach carbonneutral behavior change by 2025 . Let's be the change and foster change.
Jasia (Jayne) Steinmetz, $R D, C D$

President, Society for Nutrition Education and Behavior

\section{REFERENCES}

1. IPCC. 2021: Summary for Policymakers. In: Masson-Delmotte V, Zhai P, Pirani A, Connors SL, Péan C, Berger S, Caud N, Chen Y, Goldfarb L, Gomis MI, Huang M, Leitzell K, Lonnoy E, Matthews JBR, Maycock TK, Waterfield T, Yelekçi O, Yu R, Zhou B, eds. Climate Change 2021: The Physical Science Basis. Contribution of Working Group I to the Sixth Assessment Report of the Intergovernmental Panel on Climate Change. Cambridge University Press. In Press.

2. IPCC. IPCC Press Conference, August 9, 2021. https://www.youtube.com/watch? $\mathrm{v}=\mathrm{z} 149 \mathrm{vLKn} 9 \mathrm{~d} 8$. Accessed August 17, 2021.
3. Meerow S, Woodrow S. Seven principles of strong climate change planning. J Am Plann Assoc. 2020;86:39-46.

4. Rosenzweig C, Mbow C, Barioni L, et al. Climate change responses benefit from a global food system approach. Nat Food. 2020;1:94-97.

5. FAO. The Future of Food and Agriculture Trends and Challenges. Food and Agriculture Organization of the United $\mathrm{Na}$ tions; 2017. http://www.fao.org/3/ai6583e.pdf. Accessed August 23, 2021.

6. Boehm R, Wilde PE, Ver Ploeg M, Costello C, Cash SB. A comprehensive life cycle assessment of greenhouse gas emission from U.S. household food choices. Food Policy. 2018;29:67-76.

7. Clark MA, Domingo NGG, Colgan K, Thakrar SK, et al. Global food system emissions could preclude achieving the $1.5^{\circ}$ and $2^{\circ} \mathrm{C}$ climate change targets. Science. 2020;370:705-708. 